Commun. Fac Sci. Univ. Ank. Ser. A1 Math. Stat.

Volume 68, Number 2, Pages 1611-1620(2019)

DOI: $10.31801 /$ cfsuasmas. 546583

ISSN 1303-5991 E-ISSN 2618-6470

http://communications.science.ankara.edu.tr/index.php?series=A1

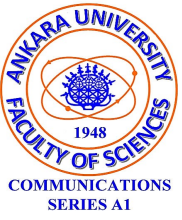

\title{
A NEW RESULT FOR WEIGHTED ARITHMETIC MEAN SUMMABILITY FACTORS OF INFINITE SERIES INVOLVING ALMOST INCREASING SEQUENCES
}

\author{
ŞEBNEM YILDIZ
}

\begin{abstract}
In this paper, a known theorem dealing with weighted mean summability methods of non-decreasing sequences has been generalized for $\left|A, p_{n} ; \delta\right|_{k}$ summability factors of almost increasing sequences. Also, some new results have been obtained concerning $\left|\bar{N}, p_{n}\right|_{k},\left|\bar{N}, p_{n} ; \delta\right|_{k}$ and $|C, 1 ; \delta|_{k}$ summability factors.
\end{abstract}

\section{INTRODUCTION}

Let $\sum a_{n}$ be a given infinite series with the partial sums $\left(s_{n}\right)$. We denote $u_{n}^{\alpha}$ the nth Cesàro mean of order $\alpha$, with $\alpha>-1$, of the sequence $\left(s_{n}\right)$, that is (see [9]),

$$
u_{n}^{\alpha}=\frac{1}{A_{n}^{\alpha}} \sum_{v=0}^{n} A_{n-v}^{\alpha-1} s_{v}
$$

where

$$
A_{n}^{\alpha}=\frac{(\alpha+1)(\alpha+2) \ldots(\alpha+n)}{n !}=O\left(n^{\alpha}\right), \quad A_{-n}^{\alpha}=0 \quad \text { for } \quad n>0 .
$$

A series $\sum a_{n}$ is said to be summable $|C, \alpha ; \delta|_{k}, k \geq 1$ and $\delta \geq 0$, if (see [10]),

$$
\sum_{n=1}^{\infty} n^{\delta k+k-1}\left|u_{n}^{\alpha}-u_{n-1}^{\alpha}\right|^{k}<\infty
$$

If we take $\delta=0$, then we have $|C, \alpha|_{k}$ summability (see [12]).

Let $\left(p_{n}\right)$ be a sequence of positive numbers such that

$$
P_{n}=\sum_{v=0}^{n} p_{v} \rightarrow \infty \quad \text { as } \quad n \rightarrow \infty, \quad\left(P_{-i}=p_{-i}=0, \quad i \geq 1\right)
$$

Received by the editors: July 31, 2018; Accepted: January 15, 2019.

2010 Mathematics Subject Classification. Primary 26D15, 40D15; Secondary 40F05, 40G99.

Key words and phrases. Riesz mean, absolute summability, infinite series.

Submitted via 2nd International Conference of Mathematical Sciences (ICMS 2018). 
The sequence-to-sequence transformation

$$
w_{n}=\frac{1}{P_{n}} \sum_{v=0}^{n} p_{v} s_{v}
$$

defines the sequence $\left(w_{n}\right)$ of the weighted arithmetic mean or simply the $\left(\bar{N}, p_{n}\right)$ mean of the sequence $\left(s_{n}\right)$ generated by the sequence of coefficients $\left(p_{n}\right)$ (see [11]). The $\left(\bar{N}, p_{n}\right)$ mean of $\left(s_{n}\right)$ reduces to the Cesàro mean $(C, 1)$ when $\left(p_{n}\right)=1$; to the logarithmic mean $(\ell, 1)$ when $\left(p_{n}\right)=\frac{1}{n+1}$ [17]. $\left(\bar{N}, p_{n}\right)$ means were used in many applications of summability theory such as Tauberian and Korovkin type- theorems (see e.g. [18, [19] and [2]).

The series $\sum a_{n}$ is said to be summable $\left|\bar{N}, p_{n} ; \delta\right|_{k}, k \geq 1$ and $\delta \geq 0$, if (see [5]),

$$
\sum_{n=1}^{\infty}\left(\frac{P_{n}}{p_{n}}\right)^{\delta k+k-1}\left|\Delta w_{n-1}\right|^{k}<\infty
$$

where

$$
\Delta w_{n-1}=-\frac{p_{n}}{P_{n} P_{n-1}} \sum_{v=1}^{n} P_{v-1} a_{v}, \quad n \geq 1 .
$$

In the special case if we take $\delta=0$, we have $\left|\bar{N}, p_{n}\right|_{k}$ summability (see [3]). When $p_{n}=1$ for all values of $n,\left|\bar{N}, p_{n} ; \delta\right|_{k}$ summability is the same as $|C, 1 ; \delta|_{k}$ summability. Also if we take $\delta=0$ and $k=1$, then we have $\left|\bar{N}, p_{n}\right|$ summability.

Let $A=\left(a_{n v}\right)$ be a normal matrix. i.e., a lower triangular matrix of nonzero diagonal entries. Given a normal matrix $A=\left(a_{n v}\right)$, we associate two lower semimatrices $\bar{A}=\left(\bar{a}_{n v}\right)$ and $\hat{A}=\left(\hat{a}_{n v}\right)$ as follows:

$$
\bar{a}_{n v}=\sum_{i=v}^{n} a_{n i}, \quad n, v=0,1, \ldots
$$

and

$$
\hat{a}_{00}=\bar{a}_{00}=a_{00}, \quad \hat{a}_{n v}=\bar{a}_{n v}-\bar{a}_{n-1, v}, \quad n=1,2, \ldots
$$

Then $A$ defines the sequence-to-sequence transformation, mapping the sequence $s=\left(s_{n}\right)$ to $A s=\left(A_{n}(s)\right)$, where

$$
A_{n}(s)=\sum_{v=0}^{n} a_{n v} s_{v}, \quad n=0,1, \ldots
$$

It may be noted that $\bar{A}$ and $\hat{A}$ are the well-known matrices of series-to-sequence and series-to-series transformations, respectively. Then, we have

$$
A_{n}(s)=\sum_{v=0}^{n} a_{n v} s_{v}=\sum_{v=0}^{n} a_{n v} \sum_{i=0}^{v} a_{i}=\sum_{i=0}^{n} a_{i} \sum_{v=i}^{n} a_{n v}
$$




$$
=\sum_{i=0}^{n} a_{i} \bar{a}_{n i}=\sum_{v=0}^{n} \bar{a}_{n v} a_{v} .
$$

Since $\quad \bar{a}_{n-1, n}=\sum_{i=n}^{n-1} a_{n-1, i}=0$,

$$
\begin{aligned}
\bar{\Delta} A_{n}(s) & =A_{n}(s)-A_{n-1}(s)=\sum_{v=0}^{n} \bar{a}_{n v} a_{v}-\sum_{v=0}^{n-1} \bar{a}_{n-1, v} a_{v} \\
& =\sum_{v=0}^{n}\left(\bar{a}_{n v}-\bar{a}_{n-1, v}\right) a_{v}+\bar{a}_{n-1, n} a_{n}=\sum_{v=0}^{n} \hat{a}_{n v} a_{v} .
\end{aligned}
$$

The series $\sum a_{n}$ is said to be summable $\left|A, p_{n} ; \delta\right|_{k}, k \geq 1$ and $\delta \geq 0$, if (see [16])

$$
\sum_{n=1}^{\infty}\left(\frac{P_{n}}{p_{n}}\right)^{\delta k+k-1}\left|\bar{\Delta} A_{n}(s)\right|^{k}<\infty
$$

where

$$
\Delta A_{n}(s)=A_{n}(s)-A_{n+1}(s), \quad \text { and } \quad \bar{\Delta} A_{n}(s)=A_{n}(s)-A_{n-1}(s) .
$$

By a weighted mean matrix we state

$$
a_{n v}= \begin{cases}\frac{p_{v}}{P_{n}}, & 0 \leq v \leq \mathrm{n} \\ 0 & v>n\end{cases}
$$

where $\left(p_{n}\right)$ is a sequence of positive numbers with $P_{n}=p_{0}+p_{1}+p_{2}+\ldots+p_{n} \rightarrow \infty$ as $n \rightarrow \infty$.

If we take $\delta=0$, then $\left|A, p_{n} ; \delta\right|_{k}$ summability is the same as $\left|A, p_{n}\right|_{k}$ summability (see [20]) and if we take $\delta=0$ and $a_{n v}=\frac{p_{v}}{P_{n}}$, then $\left|A, p_{n} ; \delta\right|_{k}$ summability is the same as $\left|\bar{N}, p_{n}\right|_{k}$ summability. Also, if we take $\delta=0, a_{n v}=\frac{p_{v}}{P_{n}}$ and $p_{n}=1$ for all $n$, then $\left|A, p_{n} ; \delta\right|_{k}$ summability is the same as $|C, 1|_{k}$ summability.

\section{The Known Results}

Quite recently, Bor has proved the following theorems concerning on weighted arithmetic mean summability factors of infinite series.

Theorem 1. 4] Let $\left(X_{n}\right)$ be a positive non-decreasing sequence and suppose that there exists sequences $\left(\beta_{n}\right)$ and $\left(\lambda_{n}\right)$ such that

$$
\begin{aligned}
& \left|\Delta \lambda_{n}\right| \leq \beta_{n}, \\
& \beta_{n} \rightarrow 0 \quad \text { as } \quad n \rightarrow \infty \\
& \sum_{n=1}^{\infty} n\left|\Delta \beta_{n}\right| X_{n}<\infty, \\
& \left|\lambda_{n}\right| X_{n}=O(1) .
\end{aligned}
$$


If

$$
\sum_{n=1}^{m} \frac{\left|s_{n}\right|^{k}}{n}=O\left(X_{m}\right) \quad \text { as } \quad m \rightarrow \infty,
$$

and $\left(p_{n}\right)$ is a sequence that

$$
\begin{aligned}
& P_{n}=O\left(n p_{n}\right), \\
& P_{n} \Delta p_{n}=O\left(p_{n} p_{n+1}\right),
\end{aligned}
$$

then the series $\sum a_{n} \frac{P_{n} \lambda_{n}}{n p_{n}}$ is summable $\left|\bar{N}, p_{n}\right|_{k}, k \geq 1$.

Theorem 2. 6] Let $\left(X_{n}\right)$ be a positive non-decreasing sequence. If the sequences $\left(X_{n}\right),\left(\beta_{n}\right),\left(\lambda_{n}\right),\left(p_{n}\right)$ satisfy the conditions (14)-(17), (19)-(20) of Theorem 1, and

$$
\begin{aligned}
\sum_{n=1}^{m}\left(\frac{P_{n}}{p_{n}}\right)^{\delta k} \frac{\left|s_{n}\right|^{k}}{n} & =O\left(X_{m}\right) \quad \text { as } \quad m \rightarrow \infty, \\
\sum_{n=v+1}^{m+1}\left(\frac{P_{n}}{p_{n}}\right)^{\delta k-1} \frac{1}{P_{n-1}} & =O\left(\left(\frac{P_{v}}{p_{v}}\right)^{\delta k} \frac{1}{P_{v}}\right) \text { as } m \rightarrow \infty,
\end{aligned}
$$

then the series $\sum a_{n} \frac{P_{n} \lambda_{n}}{n p_{n}}$ is summable $\left|\bar{N}, p_{n} ; \delta\right|_{k}, k \geq 1$ and $0 \leq \delta<1 / k$.

Theorem 3. 7] Let $\left(X_{n}\right)$ be a positive non-decreasing sequence. If the sequences $\left(X_{n}\right),\left(\beta_{n}\right),\left(\lambda_{n}\right)$, and $\left(p_{n}\right)$ satisfy the conditions (14)-(17), (19)-(20) of Theorem 1 , and

$$
\sum_{n=1}^{m} \frac{\left|s_{n}\right|^{k}}{n X_{n}^{k-1}}=O\left(X_{m}\right) \quad \text { as } \quad m \rightarrow \infty,
$$

then the series $\sum a_{n} \frac{P_{n} \lambda_{n}}{n p_{n}}$ is summable $\left|\bar{N}, p_{n} ; \delta\right|_{k}, k \geq 1$ and $0 \leq \delta<1 / k$.

Theorem 4. [7 Let $\left(X_{n}\right)$ be a positive non-decreasing sequence. If the sequences $\left(X_{n}\right),\left(\beta_{n}\right),\left(\lambda_{n}\right)$, and $\left(p_{n}\right)$ satisfy the conditions (14)-(17), (19)-(20) of Theorem 1 , and

$$
\sum_{n=1}^{m} \frac{\left|s_{n}\right|^{k}}{n X_{n}^{k-1}}=O\left(X_{m}\right) \quad \text { as } \quad m \rightarrow \infty,
$$

then the series $\sum a_{n} \frac{P_{n} \lambda_{n}}{n p_{n}}$ is summable $\left|\bar{N}, p_{n}\right|_{k}, k \geq 1$.

Theorem 5. 8 Let $\left(X_{n}\right)$ be a positive non-decreasing sequence. If the sequences $\left(X_{n}\right),\left(\beta_{n}\right),\left(\lambda_{n}\right)$, and $\left(p_{n}\right)$ satisfy the conditions (14)-(17), (19)-(20) of Theorem 1, condition (22) of Theorem 2, and

$$
\sum_{n=1}^{m}\left(\frac{P_{n}}{p_{n}}\right)^{\delta k} \frac{\left|s_{n}\right|^{k}}{n X_{n}^{k-1}}=O\left(X_{m}\right) \quad \text { as } \quad m \rightarrow \infty,
$$


then the series $\sum a_{n} \frac{P_{n} \lambda_{n}}{n p_{n}}$ is summable $\left|\bar{N}, p_{n} ; \delta\right|_{k}, k \geq 1,0 \leq \delta<1 / k$.

We need the following lemmas.

Lemma 6. 13] Under the conditions on $\left(X_{n}\right),\left(\beta_{n}\right)$, and $\left(\lambda_{n}\right)$ as expressed in the statement of Theorem 1, we have the following:

$$
\begin{aligned}
n X_{n} \beta_{n} & =O(1), \\
\sum_{n=1}^{\infty} \beta_{n} X_{n} & <\infty .
\end{aligned}
$$

Lemma 7. 15] If the conditions (19) and (20) of Theorem 1 are satisfied, then $\Delta\left(\frac{P_{n}}{n p_{n}}\right)=O\left(\frac{1}{n}\right)$.

Remark 8. Under the conditions on the sequence $\left(\lambda_{n}\right)$ of Theorem 1, we have that $\left(\lambda_{n}\right)$ is bounded and $\Delta \lambda_{n}=O(1 / n)$ (see [4]).

\section{The Main Results}

A positive sequence $\left(b_{n}\right)$ is said to be almost increasing if there exists a positive increasing sequence $\left(z_{n}\right)$ and two positive constants $C$ and $B$ such that $C z_{n} \leq b_{n} \leq$ $B z_{n}$ (see [1]). It is known that every increasing sequences is an almost increasing sequence but the converse need not be true. In this paper we generalize Theorem 5 to $\left|A, p_{n} ; \delta\right|_{k}$ summability method using almost increasing sequences and normal matrix instead of non-decreasing sequences and weighted mean matrix, respectively. The following our main theorem is generalized the above results concerning $\left|\bar{N}, p_{n}\right|_{k}$ and $\left|\bar{N}, p_{n} ; \delta\right|_{k}$ summability methods.

Theorem 9. 22] Let $k \geq 1$ and $0 \leq \delta<1 / k$. Let $A=\left(a_{n v}\right)$ be a positive normal matrix such that

$$
\begin{aligned}
\bar{a}_{n 0} & =1, n=0,1, \ldots, \\
a_{n-1, v} & \geq a_{n v}, \text { for } n \geq v+1, \\
a_{n n} & =O\left(\frac{p_{n}}{P_{n}}\right), \\
\sum_{v=1}^{n-1} a_{v v} \hat{a}_{n, v+1} & =O\left(a_{n n}\right), \\
\sum_{n=v+1}^{m+1}\left(\frac{P_{n}}{p_{n}}\right)^{\delta k}\left|\Delta_{v}\left(\hat{a}_{n v}\right)\right| & =O\left\{\left(\frac{P_{v}}{p_{v}}\right)^{\delta k-1}\right\} \text { as } m \rightarrow \infty, \\
\sum_{n=v+1}^{m+1}\left(\frac{P_{n}}{p_{n}}\right)^{\delta k}\left|\hat{a}_{n, v+1}\right| & =O\left\{\left(\frac{P_{v}}{p_{v}}\right)^{\delta k}\right\} \text { as } m \rightarrow \infty .
\end{aligned}
$$


Let $\left(X_{n}\right)$ be an almost increasing sequence. If the sequences $\left(X_{n}\right),\left(\beta_{n}\right),\left(\lambda_{n}\right)$, and $\left(p_{n}\right)$ satisfy all the conditions of Theorem 5 , then the series $\sum a_{n} \frac{P_{n} \lambda_{n}}{n p_{n}}$ is summable $\left|A, p_{n} ; \delta\right|_{k}, k \geq 1,0 \leq \delta<1 / k$.

\section{Proof of Theorem 9}

Proof. Let $\left(V_{n}\right)$ denotes the A-transform of the series $\sum a_{n} \frac{P_{n} \lambda_{n}}{n p_{n}}$. Then, by the definition, we have that

$$
\bar{\Delta} V_{n}=\sum_{v=1}^{n} \hat{a}_{n v} a_{v} \frac{P_{v} \lambda_{v}}{v p_{v}} .
$$

Applying Abel's transformation to this sum, we have that

$$
\begin{gathered}
\bar{\Delta} V_{n}=\sum_{v=1}^{n-1} \Delta_{v}\left(\frac{\hat{a}_{n v} P_{v} \lambda_{v}}{v p_{v}}\right) \sum_{r=1}^{v} a_{r}+\frac{\hat{a}_{n n} P_{n} \lambda_{n}}{n p_{n}} \sum_{r=1}^{n} a_{r} \\
\bar{\Delta} V_{n}=\sum_{v=1}^{n-1} \Delta_{v}\left(\frac{\hat{a}_{n v} P_{v} \lambda_{v}}{v p_{v}}\right) s_{v}+\frac{\hat{a}_{n n} P_{n} \lambda_{n}}{n p_{n}} s_{n}, \\
\bar{\Delta} V_{n}=\frac{a_{n n} P_{n} \lambda_{n}}{n p_{n}} s_{n}+\sum_{v=1}^{n-1} \frac{P_{v} \lambda_{v}}{v p_{v}} \Delta_{v}\left(\hat{a}_{n v}\right) s_{v}+\sum_{v=1}^{n-1} \hat{a}_{n, v+1} \lambda_{v} \Delta\left(\frac{P_{v}}{v p_{v}}\right) s_{v} \\
+\sum_{v=1}^{n-1} \hat{a}_{n, v+1} \frac{P_{v+1}}{(v+1) p_{v+1}} \Delta \lambda_{v} s_{v} \\
\bar{\Delta} V_{n}=V_{n, 1}+V_{n, 2}+V_{n, 3}+V_{n, 4} .
\end{gathered}
$$

To complete the proof of Theorem 9, by Minkowski inequality, it is sufficient to show that

$$
\sum_{n=1}^{\infty}\left(\frac{P_{n}}{p_{n}}\right)^{\delta k+k-1}\left|V_{n, r}\right|^{k}<\infty, \quad \text { for } \quad r=1,2,3,4 .
$$

First, by applying Hölder's inequality with indices $k$ and $k^{\prime}$, where $k>1$ and $\frac{1}{k}+\frac{1}{k^{\prime}}=1$, we have that

$$
\begin{aligned}
& \sum_{n=1}^{m}\left(\frac{P_{n}}{p_{n}}\right)^{\delta k+k-1}\left|V_{n, 1}\right|^{k} \leq \sum_{n=1}^{m}\left(\frac{P_{n}}{p_{n}}\right)^{\delta k+k-1} a_{n n}^{k}\left(\frac{P_{n}}{p_{n}}\right)^{k}\left|\lambda_{n}\right|^{k} \frac{\left|s_{n}\right|^{k}}{n^{k}} \\
& =O(1) \sum_{n=1}^{m}\left(\frac{P_{n}}{p_{n}}\right)^{\delta k+k-1}\left|\lambda_{n}\right|^{k} \frac{\left|s_{n}\right|^{k}}{n^{k}}=O(1) \sum_{n=1}^{m}\left(\frac{P_{n}}{p_{n}}\right)^{\delta k} \frac{n^{k-1}}{n^{k}}\left|\lambda_{n}\right|^{k-1}\left|\lambda_{n}\right|\left|s_{n}\right|^{k} \\
& =O(1) \sum_{n=1}^{m}\left(\frac{P_{n}}{p_{n}}\right)^{\delta k} \frac{1}{n} \frac{1}{X_{n}^{k-1}}\left|\lambda_{n}\right|\left|s_{n}\right|^{k} \\
& =O(1) \sum_{n=1}^{m-1} \Delta\left|\lambda_{n}\right| \sum_{v=1}^{n}\left(\frac{P_{v}}{p_{v}}\right)^{\delta k} \frac{\left|s_{v}\right|^{k}}{v X_{v}^{k-1}}+O(1)\left|\lambda_{m}\right| \sum_{n=1}^{m}\left(\frac{P_{n}}{p_{n}}\right)^{\delta k} \frac{\left|s_{n}\right|^{k}}{n X_{n}^{k-1}}
\end{aligned}
$$




$$
=O(1) \sum_{n=1}^{m-1}\left|\Delta \lambda_{n}\right| X_{n}+O(1)\left|\lambda_{m}\right| X_{m}=O(1) \sum_{n=1}^{m-1} \beta_{n} X_{n}+O(1)\left|\lambda_{m}\right| X_{m}=O(1)
$$

as $m \rightarrow \infty$.By applying Hölder's inequality with indices $k$ and $k^{\prime}$, where $k>1$ and $\frac{1}{k}+\frac{1}{k^{\prime}}=1$ and as in $V_{n, 1}$, we have that

$$
\begin{aligned}
& \sum_{n=2}^{m+1}\left(\frac{P_{n}}{p_{n}}\right)^{\delta k+k-1}\left|V_{n, 2}\right|^{k}=\sum_{n=2}^{m+1}\left(\frac{P_{n}}{p_{n}}\right)^{\delta k+k-1}\left|\sum_{v=1}^{n-1} \frac{P_{v} \lambda_{v}}{v p_{v}} \Delta_{v}\left(\hat{a}_{n v}\right) s_{v}\right|^{k} \\
& \leq \sum_{n=2}^{m+1}\left(\frac{P_{n}}{p_{n}}\right)^{\delta k+k-1}\left\{\sum_{v=1}^{n-1}\left|\Delta_{v}\left(\hat{a}_{n v}\right)\right|\left|\lambda_{v}\right|^{k}\left|s_{v}\right|^{k} \frac{1}{v^{k}}\left(\frac{P_{v}}{p_{v}}\right)^{k}\right\} \times\left\{\sum_{v=1}^{n-1}\left|\Delta_{v}\left(\hat{a}_{n v}\right)\right|\right\}^{k-1} \\
& =O(1) \sum_{n=2}^{m+1}\left(\frac{P_{n}}{p_{n}}\right)^{\delta k+k-1} a_{n n}^{k-1} \sum_{v=1}^{n-1}\left|\Delta_{v}\left(\hat{a}_{n v}\right)\right|\left|\lambda_{v}\right|^{k}\left|s_{v}\right|^{k} \frac{1}{v^{k}}\left(\frac{P_{v}}{p_{v}}\right)^{k} \\
& =O(1) \sum_{v=1}^{m}\left(\frac{P_{n}}{p_{n}}\right)^{\delta k}\left|\lambda_{v}\right|^{k-1}\left|\lambda_{v}\right|\left|s_{v}\right|^{k} \frac{1}{v^{k}}\left(\frac{P_{v}}{p_{v}}\right)^{k} \sum_{n=v+1}^{m+1}\left|\Delta_{v}\left(\hat{a}_{n v}\right)\right| \\
& =O(1) \sum_{v=1}^{m}\left(\frac{P_{v}}{p_{v}}\right)^{\delta k} a_{v v} \frac{1}{X_{v}^{k-1}}\left|s_{v}\right|^{k}\left|\lambda_{v}\right| \frac{1}{v^{k}}\left(\frac{P_{v}}{p_{v}}\right)^{k} \\
& =O(1) \sum_{v=1}^{m}\left(\frac{P_{v}}{p_{v}}\right)^{\delta k} \frac{1}{X_{v}^{k-1}}\left|s_{v}\right|^{k}\left|\lambda_{v}\right| \frac{1}{v^{k}}\left(\frac{P_{v}}{p_{v}}\right)^{k-1} \\
& =O(1) \sum_{v=1}^{m}\left(\frac{P_{v}}{p_{v}}\right)^{\delta k} \frac{1}{X_{v}^{k-1}}\left|s_{v}\right|^{k}\left|\lambda_{v}\right| \frac{1}{v^{k}} v^{k-1} \\
& =O(1) \sum_{v=1}^{m}\left(\frac{P_{v}}{p_{v}}\right)^{\delta k} \frac{1}{X_{v}^{k-1}}\left|s_{v}\right|^{k}\left|\lambda_{v}\right| \frac{1}{v}=O(1) \quad \text { as } \quad m \rightarrow \infty .
\end{aligned}
$$

Also, by using conditions of Theorem 9, we obtain that

$$
\begin{aligned}
& \sum_{n=2}^{m+1}\left(\frac{P_{n}}{p_{n}}\right)^{\delta k+k-1}\left|V_{n, 3}\right|^{k}=\sum_{n=2}^{m+1}\left(\frac{P_{n}}{p_{n}}\right)^{\delta k+k-1}\left|\sum_{v=1}^{n-1} \hat{a}_{n, v+1} \Delta\left(\frac{P_{v}}{v p_{v}}\right) \lambda_{v} s_{v}\right|^{k} \\
& =O(1) \sum_{n=2}^{m+1}\left(\frac{P_{n}}{p_{n}}\right)^{\delta k+k-1}\left\{\sum_{v=1}^{n-1} a_{v v}^{1-k} \hat{a}_{n, v+1}\left|\lambda_{v}\right|^{k}\left|s_{v}\right|^{k} \frac{1}{v^{k}}\right\} \times\left\{\sum_{v=1}^{n-1} a_{v v} \hat{a}_{n, v+1}\right\}^{k-1} \\
& =O(1) \sum_{n=2}^{m+1}\left(\frac{P_{n}}{p_{n}}\right)^{\delta k+k-1} a_{n n}^{k-1} \sum_{v=1}^{n-1}\left(\frac{P_{v}}{p_{v}}\right)^{k-1} \hat{a}_{n, v+1}\left|\lambda_{v}\right|^{k}\left|s_{v}\right|^{k} \frac{1}{v^{k}} \\
& =O(1) \sum_{v=1}^{m}\left(\frac{P_{v}}{p_{v}}\right)^{\delta k}\left|\lambda_{v}\right|^{k-1}\left|\lambda_{v}\right|\left|s_{v}\right|^{k} \frac{1}{v} \sum_{n=v+1}^{m+1} \hat{a}_{n, v+1}
\end{aligned}
$$




$$
\begin{aligned}
& =O(1) \sum_{v=1}^{m}\left(\frac{P_{v}}{p_{v}}\right)^{\delta k}\left|\lambda_{v}\right|^{k-1}\left|\lambda_{v}\right|\left|s_{v}\right|^{k} \frac{1}{v} \\
& =O(1) \sum_{v=1}^{m}\left(\frac{P_{v}}{p_{v}}\right)^{\delta k} \frac{1}{X_{v}^{k-1}}\left|\lambda_{v}\right|\left|s_{v}\right|^{k} \frac{1}{v}=O(1) \quad \text { as } \quad m \rightarrow \infty .
\end{aligned}
$$

Finally, by virtue of the hypotheses of Theorem 9 , by Lemma 6 , we have $v \beta_{v}=$ $O\left(\frac{1}{X_{v}}\right)$, then

$$
\begin{aligned}
\sum_{n=2}^{m+1}\left(\frac{P_{n}}{p_{n}}\right)^{\delta k+k-1}\left|V_{n, 4}\right|^{k}=\sum_{n=2}^{m+1}\left(\frac{P_{n}}{p_{n}}\right)^{\delta k+k-1}\left|\sum_{v=1}^{n-1} \hat{a}_{n, v+1} \frac{P_{v+1}}{(v+1) p_{v+1}} \Delta \lambda_{v} s_{v}\right|^{k} \\
=O(1) \sum_{n=2}^{m+1}\left(\frac{P_{n}}{p_{n}}\right)^{\delta k+k-1}\left\{\left.\sum_{v=1}^{n-1} a_{v v}^{1-k} \hat{a}_{n, v+1}\left|\Delta \lambda_{v}\right|^{k} s_{v}\right|^{k}\right\} \times\left\{\sum_{v=1}^{n-1} a_{v v} \hat{a}_{n, v+1}\right\}^{k-1} \\
=O(1) \sum_{n=2}^{m+1}\left(\frac{P_{n}}{p_{n}}\right)^{\delta k+k-1} a_{n n}^{k-1} \sum_{v=1}^{n-1} a_{v v}^{1-k} \hat{a}_{n, v+1}\left|\Delta \lambda_{v}\right|^{k}\left|s_{v}\right|^{k} \\
=O(1) \sum_{v=1}^{m}\left(\frac{P_{v}}{p_{v}}\right)^{\delta k}\left(\frac{P_{v}}{p_{v}}\right)^{k-1}\left|s_{v}\right|^{k}\left|\Delta \lambda_{v}\right|^{k} \sum_{n=v+1}^{m+1} \hat{a}_{n, v+1} \\
=O(1) \sum_{v=1}^{m}\left(\frac{P_{v}}{p_{v}}\right)^{\delta k}\left|s_{v}\right|^{k}\left(v \beta_{v}\right)^{k-1} \beta_{v}=O(1) \sum_{v=1}^{m} v \beta_{v}\left|s_{v}\right|^{k} \frac{1}{v X_{v}^{k-1}} \\
=O(1) \sum_{v=1}^{m-1} \Delta\left(v \beta_{v}\right) \sum_{r=1}^{v}\left(\frac{P_{r}}{p_{r}}\right)^{\delta k} \frac{\left|s_{r}\right|^{k}}{r X_{r}^{k-1}}+O(1) m \beta_{m} \sum_{v=1}^{m}\left(\frac{P_{v}}{p_{v}}\right)^{\delta k} \frac{\left|s_{v}\right|^{k}}{v X_{v}^{k-1}} \\
=O(1) \sum_{v=1}^{m-1}\left|\Delta\left(v \beta_{v}\right)\right| X_{v}+O(1) m \beta_{m} X_{m}=O(1) \sum_{v=1}^{m-1}\left|(v+1) \Delta \beta_{v}-\beta_{v}\right| X_{v} \\
+O(1) m \beta_{m} X_{m} \\
=O(1) \sum_{v=1}^{m-1} v\left|\Delta \beta_{v}\right| X_{v}+O(1) \sum_{v=1}^{m-1} X_{v} \beta_{v}+O(1) m \beta_{m} X_{m}=O(1) \quad a s \quad m \rightarrow \infty
\end{aligned}
$$

This completes the proof of Theorem 9 .

Conclusion 10. If we take $\delta=0$ in Theorem 9, then Theorem 9 reduces to $\left|A, p_{n}\right|_{k}$ summability theorem (see [21]).

Let $\left(X_{n}\right)$ be a positive non-decreasing sequence. The following results have been obtained.

1. If we take $a_{n v}=\frac{p_{v}}{P_{n}}$ in Theorem 9, then Theorem 9 reduces to Theorem 5 .

2. If we take $\delta=0$ and $a_{n v}=\frac{p_{v}}{P_{n}}$ in Theorem 9, then we obtain Theorem 4 and 
if we put $\delta=0$ and $k=1$ in Theorem 5, we have a known result of Mishra and Srivastava dealing with $\left|\bar{N}, p_{n}\right|$ summability factors of infinite series (see [15]).

3. If we take $a_{n v}=\frac{p_{v}}{P_{n}}$ and $p_{n}=1$ for all values of $n$ in Theorem 9, then we obtain a known result of Mishra and Srivastava concerning the $|C, 1 ; \delta|_{k}$ summability factors of infinite series.

4. If we take $\delta=0, a_{n v}=\frac{p_{v}}{P_{n}}$ and $p_{n}=1$ for all values of $n$ in Theorem 9, then we obtain a known result of Mishra and Srivastava concerning the $|C, 1|_{k}$ summability factors of infinite series (see [14]).

\section{REFERENCES}

[1] Bari, N.K. and Stechkin, S.B., Best approximation and differential properties of two conjugate functions, Tr. Mosk. Mat. Obshch., vol. 5 (1956), 483-522.

[2] Braha, N. L., Some weighted equi-statistical convergence and Korovkin type- theorem, Res. Math., 70(34) (2014), 433-446.

[3] Bor, H., On two summability methods, Math. Proc. Camb. Philos. Soc., 97 (1985), 147-149.

[4] Bor, H., A note on $\left|\bar{N}, p_{n}\right|_{k}$ summability factors of infinite series, Indian J. Pure Appl. Math., 18 (1987), 330-336.

[5] Bor, H., On local property of $\left|\bar{N}, p_{n} ; \delta\right|_{k}$ summability of factored Fourier series, J. Math. Anal. Appl., 179 (1993), 646-649.

[6] Bor, H., A study on absolute Riesz summability factors, Rend. Circ. Mat. Palermo (2), 56 (2007) 358-368.

[7] Bor, H., Factors for absolute weighted arithmetic mean summability of infinite series, Int. J. Anal. and Appl., 14 (2) (2017), 175-179.

[8] Bor, H., On some new results for non-decreasing sequences, Tbilisi Math. J., 10 (2), (2017), 57-64.

[9] Cesàro, E., Sur la multension of absolute summability and some theorems of Littlewood and Paley, Proc. Lond. Math. Soc., 7 (1957), 113-141.

[10] Flett, T. M., Some more theorems concerning the absolute summability of Fourier series and power series, Proc.London Math. Soc., 8 (1958), 357-387.

[11] Hardy, G. H., Divergeiplication des séries, Bull. Sci. Math., 14 (1890), 114-120.

[12] Flett, T. M., On an extnt Series, Clarendon Press, Oxford 1949.

[13] Mishra, K. N., On the absolute Nörlund summability factors of infinite series, Indian J. Pure Appl. Math., 14 (1983), 40-43.

[14] Mishra, K. N. and Srivastava, R. S. L., On the absolute Cesaro summability factors of infinite series, Portugal Math., 42 (1983/84), 53-61.

[15] Mishra, K. N. and Srivastava, R. S. L., On $\left|\bar{N}, p_{n}\right|$ summability factors of infinite series, Indian J. Pure Appl. Math.,15 (1984), 651-656.

[16] Özarslan, H. S. and Öğdük, H. N., Generalizations of two theorems on absolute summability methods, Aust. J. Math. Anal. Appl. 13 (2004), 7pp.

[17] Powell, R. E. and Shah, S. M., Summability theory and its applications, Van Nostrand, London, 1972.

[18] Sezer, S. A. and Canak, I., Tauberian remainder theorems for the weighted mean method of summability, Math. Model. Anal., 19(2) (2014), 275-280.

[19] Sezer, S. A. and Canak, I., On a Tauberian theorem for the weighted mean method of summability, Kuwait J. Sci., 42(3) (2015), 1-9.

[20] Sulaiman, W. T, Inclusion theorems for absolute matrix summability methods of an infinite series, Indian J. Pure Appl. Math. 34 (11) (2003), 1547-1557. 
[21] Yildiz, Ş. A matrix application on absolute weighted arithmetic mean summability factors of infinite series, Tibilisi Math.J., (11) 2 (2018), 59-65.

[22] Yildiz, Ş. A new result on weighted arithmetic mean summability of almost increasing sequences, 2nd International Conference of Mathematical Sciences (ICMS 2018), Maltepe University,31 July 2018-6 August 2018.

Current address: Şebnem Yıldız:Kırşehir Ahi Evran University, Department of Mathematics, Arts and Sciences Faculty

E-mail address: sebnemyildiz@ahievran.edu.tr; sebnem.yildiz82@gmail.com

ORCID Address: https://orcid.org/0000-0003-3763-0308 